\title{
Traumatic Anterior Dislocation of Hip in a Child- Case Report
}

\author{
S Ahmad, MS Orth, P Devkota, MS Orth, KG Mamman, FRCS* \\ Department of Orthopaedics and Trauma Surgery, Suri Seri Begawan Hospital \\ Kuala Belait, Brunei Darussalam \\ *Department of Orthopaedics and Trauma Surgery, RIPAS Hospital, Bandar Seri Begawan, Brunei Darussalam
}

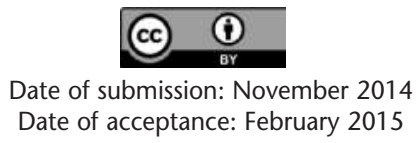

\begin{abstract}
Traumatic hip dislocation in children is relatively rare accounting for about $5 \%$ of all hip dislocations. Most of the hip dislocations seen in children are of the posterior type but the much rarer anterior and anterior-inferior (obturator) types have also been described. We present the case of an eight years old girl with an obturator type of hip dislocation following trivial trauma. She was treated with closed reduction and immobilisation in skin traction for three weeks. She was followed up closely for one year and did not develop any complications during that period.
\end{abstract}

Key Words:

Hip, anterior dislocation, child, trauma

\section{INTRODUCTION}

Traumatic hip dislocation in children is relatively a rare injury accounting for about $5 \%$ of all hip dislocations 1 . Most of the hip dislocations seen in children are of the posterior type but the much rarer anterior and anterior-inferior (obturator) types have also been described. We report the case of an eight years old girl who presented to the accident and emergency department with this rare injury following a fall.

\section{CASE REPORT}

An eight years old girl was brought to the Accident and Emergency department following a fall whilst playing with friends. She had a painfully deformed right hip with the right leg fixed in abduction and external rotation, and unable to bear weight.

On examination the right hip was fixed in flexion, abduction and external rotation. There was no neurological deficit and the distal pulses were palpable. The radiographs of the pelvis revealed a hip dislocation with the femoral head lying inferior to the acetabulum, in the obturator foramen (Figure 1). Under general anaesthesia, within six hours of injury, the dislocation was reduced by traction, hyperabduction, external rotation and adduction and internal rotation after the femoral head was cleared from the obturator foramen. Postreduction radiographs confirmed the femoral head in the acetabulum without any fractures (Figure 2). The right lower limb was immobilized in skin traction for three weeks.

On discharge from the hospital after three weeks, the patient had painless, full range movements of the left hip. She was discharged home with axillary crutches to allow weight bearing as tolerated. Radiographs of the pelvis showed a well reduced right femoral head. The patient was reviewed at three and six months.. She had a painless right hip and was ambulating unassisted full weight bearing. Radiographs showed a well reduced femoral head with no signs of avascular necrosis (AVN).

At one year follow-up, the patient had painless full-range movements in the right hip with no signs of AVN on plain radiographs as well as on magnetic resonance image (Figure 3).

\section{DISCUSSION}

Traumatic hip dislocation in children is relatively a rare injury accounting for about $5 \%$ of all hip dislocations. Most of the hip dislocations seen in children are of the posterior type but the much rare anterior type has also been described accounting for about $5 \%$ to $10 \%$ of all paediatric hip dislocations ${ }^{2,3}$, The anterior-inferior (obturator) type has been reported in less than five cases in the English literature ${ }^{1}$. This dislocation can be caused by high or low energy trauma 2,3 . Complications include associated fractures $(40 \%)^{4}$, neurological and vascular compromise $(25 \%)^{3}$, AVN $(10 \%)^{3,4,5}$ and articular cartilage injury $(6 \%)^{2}$. 


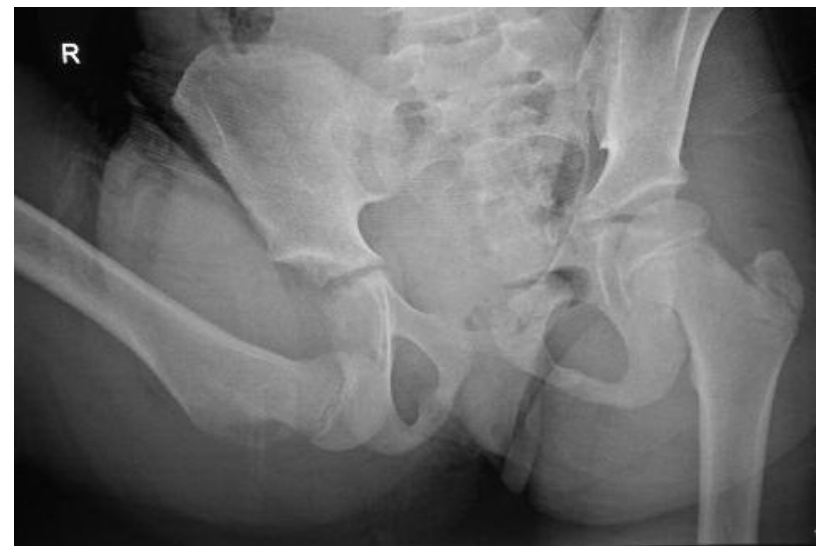

Fig. 1: Plain radiograph of the child showing anterior obturator dislocation of right hip.

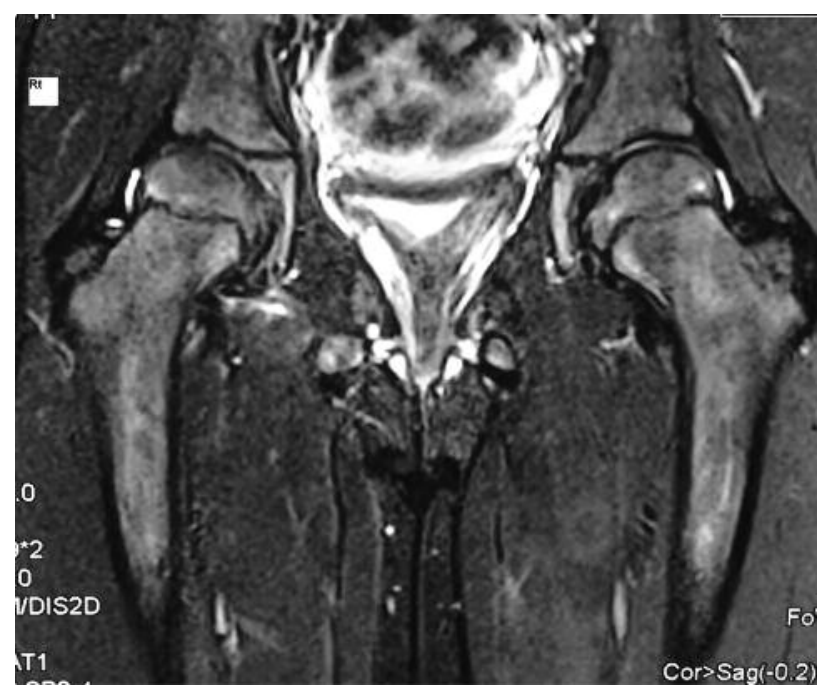

Fig. 3: Magnetic resonance image (MRI) of the right hip after one year showed no evidence of avascular necrosis (AVN).

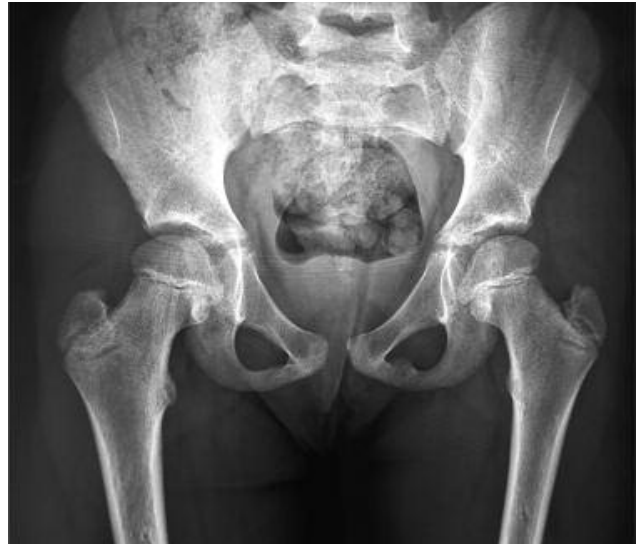

Fig. 2: Post reduction radiograph showing good reduction of the hip.

This injury should be treated as an emergency and reduced within six hours of injury, as a delayed reduction predisposes to $\mathrm{AVN}^{3}$. Although the outcome of $\mathrm{AVN}$ in children is similar to that in adults, the complication is more devastating in children as treatment options for AVN present a challenge in children ${ }^{3}$. To reduce the risk of $\mathrm{AVN}$, reduction should be carried out as early as possible with sufficient sedation and analgesia to lower the risk for physeal injury³.

After an early reduction and activity modification our patient recovered well with no signs of AVN or physeal injury after one year and parents were advised for an annual follow-up of the child with MRI scan and modification of lifestyle.

\section{REFERENCES}

1. Avery DM 3rd, Carolan GF. Traumatic obturator hip disloation in a 9-year old boy. Am J Orthop 2013; 42(9): 81-3.

2. Vialle R, Odent T, Pannier S, Pauthier F, Laumonier F, Glorian C. Traumatic hip dislocation in childhood. J Pediatr Orthop 2005; 25(2): 138-44.

3. Herrera-Soto JA, Price CT. Traumatic hip dislocations in children and adolescent: pitfalls and complications. J Am Acad Orthop Surg 2009; 17(1): 15-21.

4. Mehlman CT, Hubbard GW, Crawford AH, Roy DR, Wall EJ. Traumatic hip dislocation in children. Clin Orthop Relat Reser 2000; 376: 68-79.

5. Notzli HP, Siebenrock KA, Hempfing A, Ramseier LE, Ganz R. Perfusion of the femoral head during surgical dislocation of the hip. Monitoring by laser Doppler flowmetry. J Bone Joint Surg Br 2002; 84(2): 300-4. 